\title{
Myocardial ischemia and previous infarction contribute to left ventricular dyssynchrony in patients with coronary artery disease
}

\author{
Hanna Hämäläinen, MD, ${ }^{\mathrm{a}}$ Alisa Corovai, B. M., ${ }^{\mathrm{a}}$ Jussi Laitinen, M.Sc, ${ }^{\mathrm{a}}$ \\ Tiina M. Laitinen, PhD, ${ }^{a}$ Marja Hedman, MD, PhD, ,c Antti Hedman, MD, PhD, ${ }^{d}$ \\ Antti Kivelä, $M D{ }^{d}$ and Tomi P. Laitinen, $M D, P h D^{a, b}$ \\ a Department of Clinical Physiology and Nuclear Medicine, Kuopio University Hospital, Kuopio, \\ KYS, Finland \\ b Institute of Clinical Medicine, University of Eastern Finland, Kuopio, Finland \\ c Department of Cardiothoracic Surgery, Kuopio University Hospital, Kuopio, Finland \\ d Heart Center, Kuopio University Hospital, Kuopio, Finland
}

Received Dec 31, 2019; accepted Jul 14, 2020

doi: $10.1007 / \mathrm{s} 12350-020-02316-9$

Aims. The aim of this study was to characterize determinants of left ventricular mechanical dyssynchrony (LVMD) in patients with coronary artery disease (CAD).

Methods. Medical records and results of myocardial perfusion SPECT/CT studies were evaluated in 326 patients with previously diagnosed CAD. LVMD was assessed with the phase analysis of ECG-gated myocardial SPECT. Dyssynchrony was described with phase histogram bandwidth (PHBW), standard deviation (PHSD) or entropy (PHE) values above limit of the highest normal.

Results. Prevalence of LVMD was $29 \%$ in CAD patients. Size of the infarction scar and ischemia extent correlated significantly with PHBW, PHSD and PHE $(P<0.001$ for all). Independent predictors of LVMD were myocardial infarction scar $(P=0.004)$, ischemia extent $(P=0.003)$, and $Q R S$ duration $(P=\mathbf{0 . 0 0 3})$. Previous percutaneous coronary intervention and coronary artery bypass grafting did not independently predict dyssynchrony.

Conclusions. Almost one-third of CAD patients had significant LVMD. Dyssynchrony was associated with earlier myocardial infarction and presence of myocardial ischemia. Previous percutaneous coronary intervention and coronary artery bypass grafting did not independently predict dyssynchrony. (J Nucl Cardiol 2021;28:3010-20.)

Key Words: Heart failure • gated SPECT • dyssynchrony • CAD

Electronic supplementary material The online version of this article (https://doi.org/10.1007/s12350-020-02316-9) contains supplementary material, which is available to authorized users.

The authors of this article have provided a PowerPoint file, available for download at SpringerLink, which summarises the contents of the paper and is free for re-use at meetings and presentations. Search for the article DOI on SpringerLink.com.

Funding This study was financially supported by Finnish Cultural Foundation, Paavo Nurmi Foundation, The Finnish Medical Foundation, Finnish Foundation for Cardiovascular Research, Finnish Cardiac Society and Specified governments transfers to Kuopio University Hospital (5031349).
Reprint requests: Hanna Hämäläinen, MD, Department of Clinical Physiology and Nuclear Medicine, Kuopio University Hospital, PO BOX 100Kuopio70029, KYS , Finland; hanna.hamalainen@kuh.fi $1071-3581 / \$ 34.00$

Copyright (C) 2020 The Author(s) 


\begin{tabular}{|ll|}
\hline Abbreviations & \\
CAD & Coronary artery disease \\
LVMD & Left ventricular \\
& dyssynchrony \\
PHBW & Phase histogram bandwidth \\
PHSD & Phase histogram standard deviation \\
PHE & Phase histogram entropy \\
PCI & Percutaneous coronary intervention \\
CABG & Coronary artery bypass graft \\
LV & Left ventricle \\
EF & Ejection fraction \\
SPECT & Single-photon emission computed \\
& tomography \\
\hline
\end{tabular}

See related editorial, pp. 3021-3024 INTRODUCTION

Understanding of mechanisms behind development of heart failure progression and therefore, characterization of risk factors predicting left ventricular (LV) dysfunction is important in patients with incipient heart failure. Left ventricular mechanical dyssynchrony (LVMD), asynchronous and dyscoordinate contraction of the $\mathrm{LV}$, has found to be a significant determinant of systolic dysfunction in patients with heart failure. ${ }^{1}$ It limits the LV function and reduces cardiac output.,

Myocardial perfusion imaging (MPI) with ECGgated single-photon emission computed tomography (SPECT) is widely used to assess possible myocardial ischemia and to study viability of myocardium to optimize treatments of coronary artery disease (CAD) patients. ${ }^{4}$ Phase analysis combined with SPECT MPI is a quite new method and it has become a validated method observing LVMD. ${ }^{5,6}$ With phase analysis, it is possible to evaluate automatically the parameters of cardiac synchrony, with high reproducibility, without any discomfort to patient, and regardless of the examiner. ${ }^{7}$ SPECT MPI offers a way to obtain simultaneous evaluation of regional myocardial perfusion, $\mathrm{LV}$ function and volumes as well as LVMD. ${ }^{8}$

LVMD has been found to occur even without signs or symptoms of heart disease and it may help to identify patients at higher risk for progression of heart failure. ${ }^{7,8}$ The increase in LVMD may be related to multivessel CAD and the severity of coronary artery stenosis. ${ }^{8-10}$ LVMD have been seen on the first day after acute myocardial infarction and it has been reported to be present only in the infarcted myocardium or both the infarcted and non-infarcted areas. ${ }^{11,12}$ The association between LVMD, myocardial infarct scars and ischemia have earlier been studied with different methods, but there is still versatility between the studies in the determinants of LVMD in patients with CAD. ${ }^{13-16}$ Also, invasive cardiac procedures like percutaneous coronary intervention (PCI) or coronary artery bypass grafting $(\mathrm{CABG})$ are important treatments for CAD, but there is still a lack of certainty about how these procedures may contribute to LVMD.

In the present study, the aim was to investigate the relationship between LVMD and myocardial ischemia, myocardial infarction and previous invasive treatments such as PCI and CABG in a population of established CAD.

\section{METHODS}

This study was approved by the Research Ethics Committee of the Northern Savo Hospital District.

\section{Subject Selection/Data Sources}

We analyzed medical records of 1191 patients who underwent SPECT/CT examination during January 2009-May 2011 in the Department of Clinical Physiology and Nuclear Medicine, Kuopio University Hospital. Of this population, we included all patients with earlier diagnosis of CAD and with both rest and stress MPI data available to use. Additionally, the 12-lead electrocardiogram (ECG) available from the same MPI examination was the third inclusion criteria. Patients with atrial fibrillation were excluded to avoid bias to LVMD measurements. A total of 326 patients fulfilled all inclusion criteria.

We collected basic characteristics (age, gender, weight, height, body mass index), previous invasive cardiac procedures, such as PCI and CABG, chronic underlying diseases, medications and history of smoking from the medical records. In addition, we analyzed the ECG recordings and MPI data and retrospectively performed the phase analysis. All the information was collected and converted to a single, non-identifiable data set. We verified the whole population as a CAD group and divided the CAD group for subgroups to examine the effects of ischemia or infarct scars. Patients who did not exceed the reference values of phase histogram measurements were defined as non-LVMD subgroup $(\mathrm{n}=232)$ and those who exceeded belonged to LVMD subgroup $(n=94)$. In addition to that, patients with summed rest score (SRS) points two or more in MPI (described later) were classified into infarct scar subgroup $(\mathrm{n}=131)$ and ischemia\% three or more were classified into ischemia subgroup $(n=244)$. For more precise selection, we studied the effects of location of myocardial infarct scar and the location of myocardial ischemia based on the coronary artery areas to find out, whether there is correlation between increased LVMD and the location of the infarct scar or the ischemic area in myocardium.

Finally, an additional reference group of 52 patients without diagnosed cardiac diseases and normal myocardial perfusion in SPECT imaging was shown in electronic supplementary material in comparison with CAD group. The selection criteria and formation of the reference group is described in details in our previous work. ${ }^{22}$ 


\section{Myocardial Perfusion Imaging Protocol}

Patients were instructed to avoid caffeine-containing beverages and medications for 24 hours before MPI. Adenosine stress MPI with 99m-Tc-tetrofosmin was performed using 1-day protocol and all patients were studied with both stress and rest phases. At stress phase, adenosine (at rates of $140 \mu \mathrm{g} \cdot \mathrm{kg} \cdot \mathrm{min}$ and occasionally due to adenosine-related symptoms at 98 or $70 \mu \mathrm{g} \cdot \mathrm{kg} \cdot \mathrm{min}$ ) was administered intravenously for 6 minutes with injection of tracer (99m-Tc-tetrofosmin, $300 \mathrm{MBq}$ ) at 4 minutes from the onset of adenosine infusion. Low-level exercise was used in combination with adenosine infusion when possible, to avoid influence of extracardial tracer accumulation. Thirty minutes after the tracer injection, ECG-gated SPECT MPI was acquired. For the rest study, patients received an intravenous injection of tracer (99 m-Tc-tetrofosmin, $700 \mathrm{MBq}$ ) and were imaged 45 minutes afterwards.

MPI scans were performed in a supine position with a dual detector SPECT/CT system (Philips Precedence; Royal Philips N.V., Amsterdam, Netherlands) with detectors in $90^{\circ}$ configurations using $180^{\circ}$ body contour orbits with 64 projections and images were acquired with $128 \times 128$ matrix size. The gated data was acquired with 16 time bins, and with 25 second per angle (patient weight $<100 \mathrm{~kg}$ ) or 30 second per angle (patient weight $>100 \mathrm{~kg}$ ). Reconstructions were made retrospectively with HERMES Hybrid Recon Cardiology (Hermes Medical Solution AB, Stockholm, Sweden). Analyses were carried out using automated QPS/QGS2012 software (Cedars-Sinai Medical Center, Los Angeles, California).

\section{Analysis of LV Perfusion}

Stress and rest images were scored according to the 17segment LV model and a five-point scale (0; normal to 4; absence tracer uptake). The summed rest score (SRS), summed stress score (SSS), and summed difference score (SDS) for perfusion were obtained automatically by software. ${ }^{17}$ SRS from zero to one was considered normal and SRS two or more was considered to represent myocardial scar and SDS two or more was considered to represent myocardial ischemia. We defined the location of an infarct by using SRS point together with coronary arterial areas as described in EANM/ESC procedural guidelines for MPI. ${ }^{4}$ We added the perfusion points in each of these 3 arterial areas (in left anterior descendens [LAD] there were 7 segments, in left circumflex [LCX] 5 and in right coronary artery [RCA] 5 segments) to find out the location of an infarct or ischemia. If there were 2 or more SRS points in LAD area and not in other areas, this was classified as LAD infarct subgroup (LCX and RCA infarct subgroups were determined accordingly). If there were 2 or more SRS points which were divided over the myocardium and not in a specific arterial area, or if there were 2 or more SRS points in two or three of the arterial areas, the case was classified into mixed infarct group.

Furthermore, co-registration of rest and stress images and voxel-by-voxel estimation of differences described by Slomka et al. was also used in quantification of ischemia percentage. ${ }^{18}$ Perfusion defect area relative to total LV area (Extent\%) was also shown. ${ }^{19}$

\section{Quantification of LV Size, Systolic Function and Mechanical Dyssynchrony}

In the QGS algorithm, the mid-myocardial LV surface was first computed and endo- and epicardial surfaces were computed subsequently. The end-diastolic volume (EDV), end-systolic volume (ESV), stroke volume (SV) and ejection fraction (EF) were calculated from the volumes determined by the endocardial surface in different time frames.

LVMD was assessed from the ECG-gated SPECT with the phase analysis method. Phase analysis detects regional count changes in 16 time bins to observe the variation in LV wall thickness throughout the cardiac cycle. This is done for 1008 spatial points covering the whole LV area. The onset of mechanical contraction is assessed for each spatial points and the information is presented as a phase histogram which describes distribution of the timing of LV regional onset of mechanical contraction as a function of the length of the R-Rinterval. ${ }^{20}$ The phase histogram reflects the heterogeneity of regional LV contraction in timing: the greater the PHBW, PHSD, and PHE are, the more dyssynchronic the contraction is. $^{21}$

The definition of having LVMD was the values of PHBW, PHSD and/or PHE above the limit of the highest normal based on our reference material $\left(\mathrm{PHBW}>63.7^{\circ}\right.$; PHSD $>26.5^{\circ}$; $\mathrm{PHE}>63.7 \%)^{22}$

\section{Statistical Analysis}

One-sample Kolmogorov-Smirnov test was used to test the normality of distribution for continuous variables. Descriptive statistics is presented as mean \pm standard deviation. In the case of continuous variables $T$ test was used to test the statistical significance of difference between two independent samples. One-way ANOVA with least significant difference post hoc algorithm was used to test the differences between the mean values of the more than two groups. For categorical variables the Chi square test or Fisher's exact test, when the expected value in each cell was less than 5, were used. Correlations were tested using Pearson's bivariate correlations.

Logistic regression analysis was performed to find out the predictors of LVMD. At first, the logistic regression model included age, sex and variables which differed between nonLVMD and LVMD subgroups and therefore were possible predictors of LVMD.

Tests were two-sided and significance was present if $P<0.05$. All statistical calculations were performed with the SPSS for Windows program (SPSS 24.0, Chicago, IL).

\section{RESULTS}

\section{Baseline Characteristics}

The characteristics of overall patient population are summarized in Table 1. CAD group included all patients with previously diagnosed CAD, and many of them also had previous myocardial infarction and previous 
Table 1. Clinical characteristics of the study population [Mean $\pm \mathrm{SD} / \mathrm{n}(\%)]$

\begin{tabular}{|c|c|c|c|}
\hline & CAD group $(n=326)$ & Non-LVMD $(\mathrm{n}=\mathbf{2 3 2})$ & LVMD (n = 94) \\
\hline \multicolumn{4}{|l|}{ Backround information } \\
\hline Age (years) & $68 \pm 10$ & $68 \pm 10$ & $69 \pm 11 \mathrm{NS}$ \\
\hline Male & $198(61 \%)$ & $133(57 \%)$ & $65(69 \%)^{*}$ \\
\hline Weight (kg) & $83 \pm 18$ & $82 \pm 17$ & $84 \pm 19$ NS \\
\hline Height $(\mathrm{cm})$ & $167 \pm 10$ & $166 \pm 9$ & $169 \pm 10$ ** \\
\hline $\mathrm{BMI}\left(\mathrm{kg} \cdot \mathrm{m}^{2}\right)$ & $29.5 \pm 5.4$ & $29.6 \pm 5$ & $29.2 \pm 5 \mathrm{NS}$ \\
\hline Systolic BP & $145 \pm 23$ & $148 \pm 23$ & $140 \pm 23 * * *$ \\
\hline Diastolic BP & $74 \pm 10$ & $74 \pm 11$ & $75 \pm 9 \mathrm{NS}$ \\
\hline \multicolumn{4}{|l|}{ Medication } \\
\hline Betablocker & $286(88 \%)$ & $205(88 \%)$ & $81(86 \%) \mathrm{NS}$ \\
\hline ACE/AT & $231(71 \%)$ & $164(71 \%)$ & $67(71 \%)$ NS \\
\hline Calcium blocker & $104(32 \%)$ & $82(35 \%)$ & $22(23 \%) *$ \\
\hline Statin & $273(84 \%)$ & $195(84 \%)$ & $78(83 \%)$ NS \\
\hline ASA & $243(75 \%)$ & $176(76 \%)$ & $67(71 \%)$ NS \\
\hline Diabetes medication & $88(27 \%)$ & $64(28 \%)$ & $24(26 \%)$ NS \\
\hline Anticoagulant & $134(41 \%)$ & $84(36 \%)$ & $50(53 \%) *$ \\
\hline Nitrate & 202 (62\%) & 146 (63\%) & $56(60 \%)$ NS \\
\hline Diuretic & 118 (36\%) & 81 (35\%) & 37 (39\%) NS \\
\hline Antiarrythmic & $4(1 \%)$ & $2(1 \%)$ & $2(2 \%) \mathrm{NS}$ \\
\hline \multicolumn{4}{|l|}{ Comorbidities } \\
\hline Diabetes & $106(33 \%)$ & $75(32 \%)$ & $31(33 \%)$ NS \\
\hline Hypertension & $250(77 \%)$ & $185(80 \%)$ & $65(69 \%)$ NS \\
\hline Kidney disease & $22(7 \%)$ & $12(5 \%)$ & $10(9 \%)$ NS \\
\hline Pulmonary disease & $90(28 \%)$ & $64(28 \%)$ & $26(28 \%) \mathrm{NS}$ \\
\hline Cancer & $37(11 \%)$ & $25(11 \%)$ & $12(13 \%) \mathrm{NS}$ \\
\hline Myocardial infarct scar (SRS $\geq 2$ ) & $131(40 \%)$ & 72 (31\%) & $59(63 \%) * * *$ \\
\hline $\mathrm{PCl}$ & 127 (39\%) & $90(39 \%)$ & 37 (39\%) NS \\
\hline CABG & $111(34 \%)$ & 71 (31\%) & $40(43 \%) *$ \\
\hline LBBB & $23(7 \%)$ & $7(3 \%)$ & $16(17 \%)$ \\
\hline
\end{tabular}

Statistical significance between non-LVMD and LVMD groups: ${ }^{*} P<0.05,{ }^{*} P<0.01,{ }^{* * *} P<0.001$

invasive cardiac procedures like PCI $(\mathrm{n}=127 ; 39 \%)$ and CABG $(\mathrm{n}=111 ; 34 \%)$. Patients with LVMD had more myocardial infarct scars $(P<0.001)$ and they were more frequently males compared to those without $\operatorname{LVMD}(P=0.048)$. PCI had been done for $39 \%$ of the patients in both LVMD and non-LVMD group $(P=0.924)$.

\section{Incidence of LV Dyssynchrony}

About one-third (29\%) of all CAD patients had LVMD. The prevalence of LVMD raised to $45 \%$ in subpopulation with infarct scar in MPI. In the subgroup of patients with earlier PCI $(n=127)$, the prevalence of LVMD was $29 \%$ and in patients with earlier CABG $(\mathrm{n}=111)$ the prevalence of LVMD was $36 \%$.
Table 2 reports the results of myocardial perfusion scores, LV volume parameters and ECG results in the all CAD patients and in non-LVMD and LVMD groups. The perfusion scores and LV volumes were statistically significantly higher and EF was lower in the LVMD group compared to non-LVMD group. SDS was comparable between the two groups whereas SRS, SSS, ischemia\% and extent\% were higher in LVMD group as compared with non-LVMD group. Furthermore, QRS duration was significantly longer in LVMD group as compared to non-LVMD group.

Patients in CAD group had statistically significantly increased amount of ischemia, larger infarction scars and LVMD as well as decreased volume parameters compared to reference group (more detailed data are shown in the electronic supplementary material). 
Table 2. Perfusion scores, volume parameters and ECG recordings in the CAD, left ventricular dyssynchrony and non-dyssynchrony groups (mean \pm SD)

\begin{tabular}{|c|c|c|c|}
\hline Parameter & $\begin{array}{c}\text { CAD } \\
n=326\end{array}$ & $\begin{array}{c}\text { Non-LVMD } \\
(\mathrm{n}=232)\end{array}$ & $\begin{array}{c}\text { LVMD } \\
(\mathrm{n}=94)\end{array}$ \\
\hline \multicolumn{4}{|l|}{ Perfusion scores } \\
\hline Ischemia\% & $4.2 \pm 2.6$ & $3.7 \pm 2.4$ & $5.2 \pm 2.9 * * *$ \\
\hline Extent\% & $6.5 \pm 9.3$ & $4.2 \pm 6.7$ & $12.1 \pm 12.1^{* * *}$ \\
\hline SDS & $2.0 \pm 2.2$ & $1.9 \pm 2.2$ & $2.3 \pm 2.3 \mathrm{NS}$ \\
\hline SRS & $3.2 \pm 5.0$ & $1.9 \pm 3.4$ & $6.3 \pm 6.6 * * *$ \\
\hline SSS & $5.4 \pm 5.8$ & $4.0 \pm 4.7$ & $8.8 \pm 6.8^{* * *}$ \\
\hline \multicolumn{4}{|l|}{ Volume parameters } \\
\hline $\mathrm{EDV}(\mathrm{ml})$ & $111.8 \pm 60.9$ & $97 \pm 47$ & $149 \pm 74^{* * *}$ \\
\hline $\mathrm{ESV}(\mathrm{ml})$ & $50.3 \pm 50.4$ & $35 \pm 31$ & $89 \pm 66 * * *$ \\
\hline $\mathrm{SV}(\mathrm{ml})$ & $61.6 \pm 18.8$ & $62 \pm 19$ & $60 \pm 19 \mathrm{NS}$ \\
\hline $\mathrm{EF}(\%)$ & $61.5 \pm 16.4$ & $68 \pm 12$ & $46 \pm 17^{* * *}$ \\
\hline \multicolumn{4}{|l|}{ ECG recordings } \\
\hline Heart rate at rest (bpm) & $95.0 \pm 23$ & $95 \pm 22$ & $94 \pm 24 \mathrm{NS}$ \\
\hline $\mathrm{QRS}(\mathrm{ms})$ & $107 \pm 25$ & $101 \pm 21$ & $121 \pm 29^{* * *}$ \\
\hline$P Q(m s)$ & $185 \pm 32$ & $182 \pm 30$ & $191 \pm 38^{*}$ \\
\hline QT (ms) & $398 \pm 36$ & $398 \pm 32$ & $400 \pm 44 \mathrm{NS}$ \\
\hline RBBB & $23(7 \%)$ & $16(7 \%)$ & $7(7 \%) \mathrm{NS}$ \\
\hline LBBB & $23(7 \%)$ & $7(3 \%)$ & $16(17 \%) * * *$ \\
\hline
\end{tabular}

SDS, Summed difference score; SRS, Summed rest score; SSS, Summed stress score; EDV, End diastolic volume; ESV, End systolic volume; SV, Stroke volume; EF, Ejection fraction

Statistical significance between non-LVMD and LVMD groups: ${ }^{*} P<0.05,{ }^{*} P<0.01,{ }^{*} * P<0.001$

Table 3 presents the phase analysis results both in the rest and stress studies in different subgroups of the patients. In the ischemia group, all phase analysis parameters were higher than in non-ischemia group $(P<0.05)$. The same was detected between the infarct and non-infarct groups. LVMD was significantly increased in stress compared to rest study in the whole CAD group $(P<0.001)$, as well as other subgroups except for in the LVMD group (NS). The change in phase analysis measurements $(\triangle \mathrm{PHBW}, \triangle \mathrm{PHSD}$ and $\Delta$ PHE) between the rest and stress studies was not statistically significant between the non-infarct and infarct patients $(P>0.05)$ or between the non-ischemic or ischemic patients $(P>0.05$ for all). In LVMD group, this change seemed to be smaller than in non-LVMD patients $(P<0.005$ for all $)$.

\section{Infarct scar, Ischemia and LV Dyssynchrony}

Figure 1 shows the relationship between infarct scar, ischemia percentage and phase histogram measurements. The correlations between the phase histogram parameters and parameters reflecting myocardial ischemia and infarct in the whole CAD population are shown in Table 4 and as scatter plot in Figure 2. Phase histogram parameters correlated statistically significantly with values reflecting infarct scar and ischemia\% of myocardium, whereas SDS was not statistically significantly correlated with phase analysis measurements.

In the subanalysis, there were 33 patients with an infarct scar only in LAD area, 21 patients with LCX areal infarct, 28 patients with RCA areal infarct and 37 patients with mixed areas infarcts. PHBW was highest in the mixed infarct group $\left(68.3 \pm 36^{\circ}\right)$ and second highest in the LAD infarct group $\left(58.8 \pm 33^{\circ}\right)$ and smallest in the LCX infarct group $\left(52.5 \pm 28^{\circ}\right)$. However, phase histogram measurements did not differ statistically significantly between the groups. Chi Square test showed no statistically significant difference in the prevalence of LVMD between these four infarct groups $(P=0.054)$. After excluding the mixed group from the analysis, there were not statistically significant difference in the prevalence of LVMD between LAD, LCX or RCA areal infarct scar $(P=0.442 \mathrm{NS})$. To elaborate the effects of vessel distribution of infarction more precisely, the regression analysis showed that if infarction (described by SRS points) was located in only 1 vessel 
distribution, it had less effect to increased LVMD ( $B=0.894, P=0.005)$ compared to 2 or more vessel distribution location $(B=1.736, P<0.001)$. Moreover, there were no statistically significant differences in phase analysis measurements in patients with detected ischemia in different arterial areas based on SDS points.

\section{Logistic Regression Analysis}

The logistic regression model took into consideration age and sex as fundamental information and as potential predictors of LVMD a set of variables thought to characterize different features of CAD (ischemia extent, infarction scar extent, CABG). Remodeling related to CAD was characterized by EDV and electrical dyssynchrony by QRS duration. According to the analysis, larger infarction extent\%, larger ischemia\%, and longer QRS duration independently predicted LVMD (Table 5). Previous CABG did not statistically significantly predict dyssynchrony. In analysis standardized with age, sex, myocardial ischemia and previous myocardial infarction, PCI or CABG did not influence on the amount of LVMD.

In additional logistic regression analysis in which EF was also added into the model, significant predictors of LVMD were male sex $(B=1.11, P=0.015)$, ischemia $(B=0.181, P=0.005)$, larger $\operatorname{EDV}(B=-$ $0.011, P=0.005)$, low EF $(B=-0.154, P<0.001)$ and longer QRS duration $(B=30.1, P<0.001)$, but not infarct scar extent $(B=-0.006, P=0.787)$.

\section{DISCUSSION}

In this study, the prevalence of LVMD was $29 \%$ among CAD patients. LVMD was associated with earlier myocardial infarction and presence of myocardial ischemia. The larger the infarct scar was, the more advanced LVMD was observed. Previous PCI and CABG did not independently predict LVMD. In CAD patients LVMD became even more evident in stress compared to rest study.

Observed LVMD prevalence in this study is about as high as in the earlier study, in which the prevalence among CAD patients was $38 \%$; it was more prevalent in patients with multivessel disease and associated with more impaired measures of LV perfusion and depressed LV systolic function but finally, it could only be predicted by ischemic burden and larger LV. ${ }^{8}$ In our study also, the patients with mixed infarct in MPI, had the highest LVMD values, which might indicate that patients with multivessel disease, are more susceptible for LVMD.

Another study detected that the overall prevalence of LVMD was $6.5 \%$ but it reached even $42 \%$ in the 

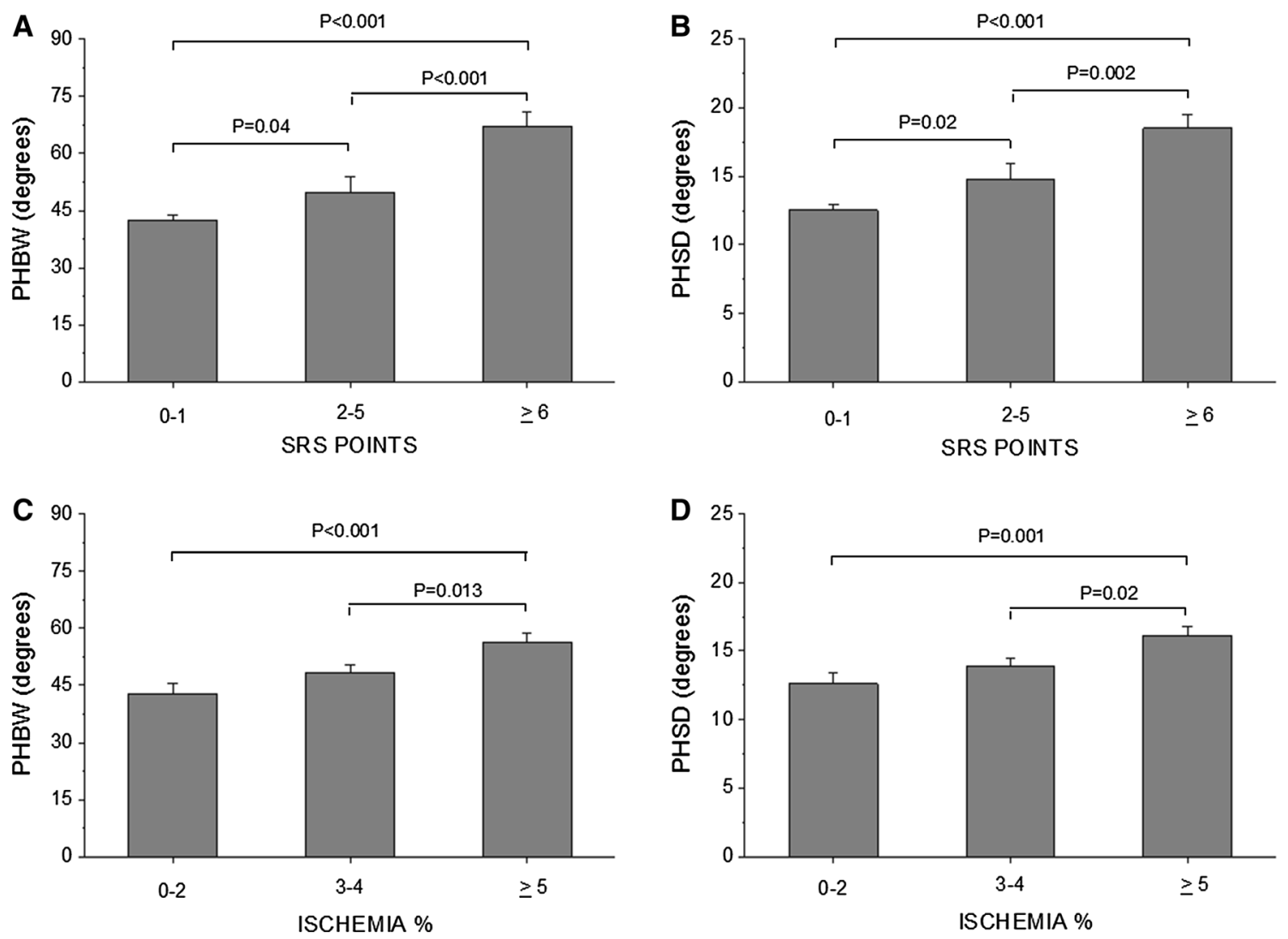

Figure 1. Relationship between A infarct scar (SRS points) and phase histogram bandwidth, B infarct scar and phase histogram standard deviation, $\mathbf{C}$ ischemia $\%$ and phase histogram bandwidth and $\mathbf{D}$ ischemia $\%$ and phase histogram standard deviation.

presence of certain risk factors (male sex and QRS > $120 \mathrm{~ms})^{7}$ They also found a significant correlation between history of CAD and LVMD; the risk for LVMD was about 5 times higher in CAD patients than those without CAD (prevalence ratio 4.44), and the previous myocardial infarct was strongly associated with LVMD, which is in line with our findings. Our study showed that the prevalence of dyssynchrony was highest among patients with previous myocardial infarct and in patients with visible infarct scars in MPI. Earlier studies have reported that LVMD was especially advanced in patients with old myocardial infarction and the extent of myocardial perfusion defect was an independent predictor for LVMD. ${ }^{23,24}$ In our study, myocardial perfusion defect measured with extent $\%$, was one of the independent predictors of LVMD. Another study used 3-dimensional echocardiography to quantify LVMD in CAD patients without regional wall motion abnormality and found out that CAD patients still demonstrated increased LVMD compared with healthy controls. $^{10}$
Logistic regression analysis of this study showed that significant predictors of LVMD were myocardial ischemia, infarction scar extent and QRS duration. In additional analysis, when EF was included in the model, significant predictors of LVMD were male sex, ischemia, larger end-diastolic volume, low ejection fraction and longer QRS duration, but not infarct scar extent. The latter model indicates how well the entire bundle of predictors predicts LVMD but it does not indicate that infarct scar would be redundant information. When including EF into the model, the analysis meets the problem of multicollinearity; patients with infarction scar have often decreased EF. ${ }^{19}$ Therefore, after adjustment of EF, the association between infarct scar and LVMD may become limited.

It has been acknowledged, that the greater the amount of LVMD was, the more perfusion defects and infarct scars were present, and myocardial infarction size was an independent predictor of LVMD. ${ }^{14,24} \mathrm{An}$ area in which the myocardial infarction locates, has also thought to matter to the amount of LVMD. The extent 
A

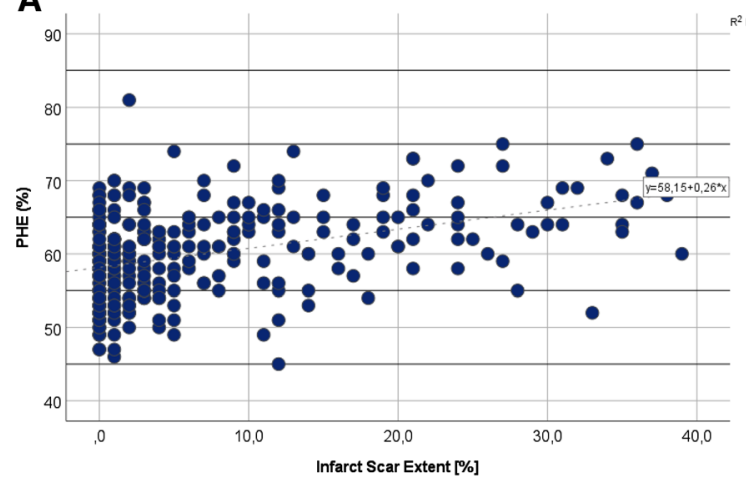

C

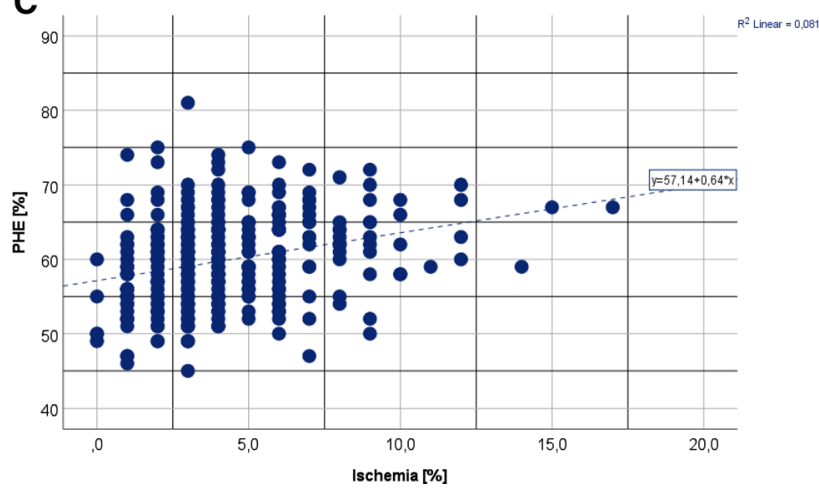

B

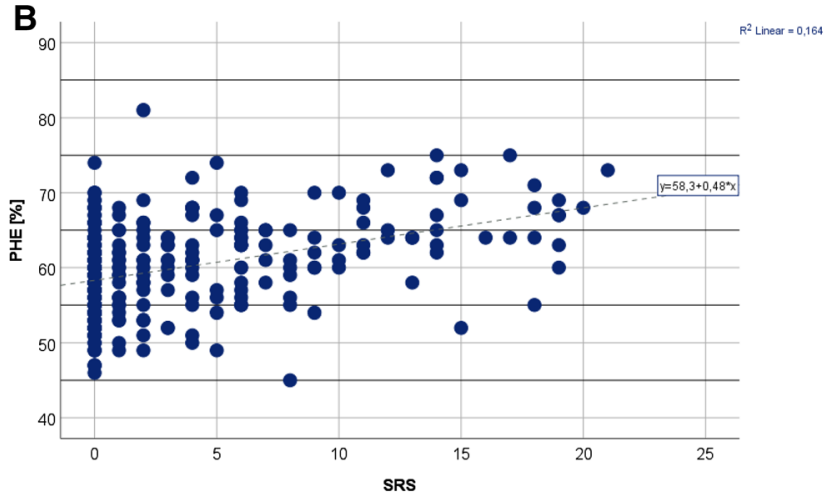

D

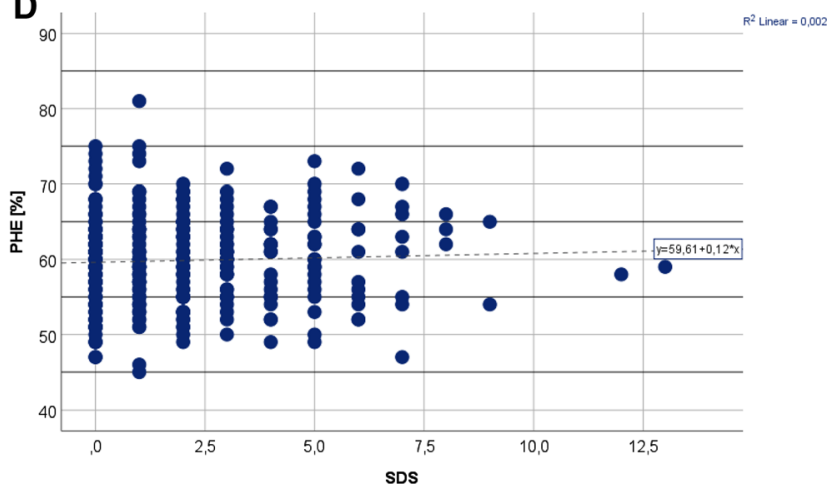

Figure 2. Correlation between phase histogram entropy (PHE) and infarction scar (A shows infarction scar as Extent $\%$ and $\mathbf{B}$ as SRS points) and myocardial ischemia ( $\mathbf{C}$ as ischemia\% and $\mathbf{D}$ as SDS points).

and severity of perfusion and regional wall motion abnormalities have found to be most prominent in the myocardial region supplied by LAD coronary artery, which may explain significantly worse post-stress LV function after anterior acute myocardial infarction. ${ }^{25} \mathrm{We}$ found statistically significant correlation between SRS as well as extent\% and increased amount of dyssynchrony. In our study, LAD infarct tended to be associated with higher dyssynchrony values compared to LCX or RCA infarct, but the difference was not statistically significant. One reason for that might be the low number of patients in each of those subgroups. Additionally, logistic regression analysis showed that if infarction scar located in 2 or more vessel territories compared to 1 vessel distribution, it had more value to increased LVMD.

In addition to previous myocardial infarction, the correlation between LVMD parameters and ischemia\% was statistically significant whereas correlation between SDS and LVMD was not. That suggests that ischemia\% might be more sensitive parameter to describe the functional significance of ischemia in the myocardium than SDS. One reason for that might be the fact that voxel-by-voxel estimation technique (Slomka et al.) compared to traditional technique has shown better ability to detect borderline-abnormal findings in patients who had coronary stenosis confirmed by coronary angiography. ${ }^{17,18}$ Therefore, it is not unexpected that we found slight, but statistically significant difference in ischemia extent but not in SDS when comparing LVMD and non-LVMD groups. LVMD has found to be associated with inducible ischemia in patients with chest pain and normal coronary arteries which supports our findings in a more clinical view. ${ }^{15}$

In multivessel CAD, more advanced LVMD have been detected at stress compared to rest, as in our study. ${ }^{9,26}$ Some studies have shown that stress-induced increasing of LVMD is observed only in patients with perfusion abnormalities, others have stated that the correlation of LVMD with reversible perfusion defects was not significant and some have showed that stressinduced ischemia increases the degree of intraventricular dyssynchrony, as discussed in our previous study. ${ }^{7,15,22,26,27}$ We found that in the pooled population and in subgroups of CAD patients with infarct, ischemia and normal LV synchrony at rest, BW, SD and entropy 
were systematically higher at stress compared to rest. This observation may be explained by the influence of ischemia burden on LV synchronicity or overestimation of LVMD due to inferior image quality related to lower injected activity in stress imaging. However, in those patients, who showed LV dyssynchrony already at rest, $\mathrm{BW}, \mathrm{SD}$ and entropy were comparable between rest and stress. We speculate that if LVMD is present already at rest further influence of stress on mechanical synchrony becomes limited.

In the present study, earlier PCI did not independently predict dyssynchrony. "Earlier PCI" in this study meant that according to medical history, PCI had been done before SPECT, but the precise timeline between PCI and SPECT was not known. Although this study did not find statistically significant association between earlier PCI and LVMD in long term, this does not prove that that PCI does not contribute to $\mathrm{LV}$

Table 4. Pearson correlation coefficients in the CAD group $(n=326)$

\begin{tabular}{lll}
\hline & Histogram BW & Histogram SD \\
\hline Extent (\%) & $0.429^{* * *}$ & $0.377^{* * *}$ \\
SRS & $0.433^{* * *}$ & $0.380^{* * *}$ \\
LAD SRS & $0.317^{* *}$ & $0.260^{* *}$ \\
LCX SRS & $0.230^{* *}$ & $0.214^{* * *}$ \\
RCA SRS & $0.263^{* *}$ & $0.239^{* * *}$ \\
Ischemia (\%) & $0.159^{* *}$ & $0.159^{* *}$ \\
SDS & $-0.018 \mathrm{NS}$ & $-0.012 \mathrm{NS}$ \\
\hline
\end{tabular}

Extent, Infarct extent (\%); SRS, summed rest score; LAD, left anterior descendens; LCX, left circumflex; RCA, Right coronary artery; Ischemia, Myocardial ischemia; SDS, Summed rest score

Statistical significance ${ }^{*} P<0.05,{ }^{*} P<0.01,{ }^{*}{ }^{*} P<0.001$ synchronicity. Earlier, even the protective value of PCI to LVMD is supported by the studies in which the most of are focused on examining the acute effects of PCI and have been done mostly with tissue Doppler echocardiography. ${ }^{28,29}$ Elective PCI as well as PCI in acute phase ST elevation infarct has found to improve LVMD significantly. ${ }^{28,29}$ LVMD within 48 hours after PCI has seemed to predict increase in LV size and deterioration of LV systolic function after 6 months follow up. ${ }^{30}$ This suggests that a significant degree of LVMD is predictive of adverse LV remodeling and may offer a possibility to identify patients at risk for LV remodeling early after infarction and to consequently emphasize intensive treatments for these patients.

In addition to PCI, it is commonly known, that CABG surgery induces paradoxical septal wall motion; about $60.5 \%$ of patients who had undergone CABG, had paradoxical septal motion quantified by SPECT. ${ }^{31}$ Use of SPECT MPI offers a way to study whether paradoxical movement is accompanied by ischemia or infarction. ${ }^{31}$ It has also been suggested, that abnormal septal motion might not be caused by ischemic insult but might occur due to an increase in anterior cardiac mobility after incision of pericardium. ${ }^{32}$ It seems that entire LV translocates anteriorly in systole after CABG, and evidence of septal infarction was not present on MRI to explain such paradoxical movement. ${ }^{33}$ Despite of this significant abnormal movement, the history of prior CABG does not seem to affect or impact LVMD indices. ${ }^{34}$ This is in line with our observations; previous CABG did not contribute to the values of dyssynchrony independently. LVMD seemed to be slightly increased in $\mathrm{CABG}$ patients compared to non-CABG patients in our extra-analysis, but this study setting could not examine the direct effects of CABG to LVMD which could be done by comparing results before and after CABG. Postoperative LVMD may reflect late reverse

Table 5. Logistic Regression predicting left ventricular mechanical dyssynchrony

\begin{tabular}{llllll}
\hline & \multicolumn{1}{c}{$\boldsymbol{B}$} & S.E. & Wald & $\boldsymbol{P}$ & OR \\
\hline Sex & 0.405 & 0.36 & 1.265 & 0.261 & 0.667 \\
Age & 0.001 & 0.016 & 0.009 & 0.925 & 0.999 \\
CABG & 0.332 & 0.305 & 1.184 & 0.277 & 1.394 \\
LBBB & 0.907 & 0.581 & 2.431 & 0.119 & 2.476 \\
Infarct(extent) & 0.054 & 0.019 & 8.403 & $0.004^{* *}$ & 1.056 \\
Ischemia\% & 0.168 & 0.057 & 8.618 & $0.003^{* *}$ & 1.183 \\
EDV(ml) & 0.007 & 0.004 & 3.649 & 0.056 & 1.007 \\
QRS (s) & 20.642 & 6.853 & 9.073 & $0.003^{* *}$ & 921639861 \\
\hline
\end{tabular}

Statistical significance; ${ }^{*} P<0.05,{ }^{*} P<0.01,{ }^{*} * P<0.001$ 
remodeling and potential of further functional improvement in patients with patent grafts and preserved perfusion reserve after CABG. ${ }^{35}$

To conclude, LVMD seems to be in close relation with CAD, myocardial infarct and ischemia. This connection expresses the importance of CAD in the pathophysiology of heart failure. The adequate prevention and treatment of CAD might prevent LVMD and thus decrease the risk for upcoming heart failure.

\section{Limitations}

The most important limitation was the retrospective nature of the study. All patients were sent to SPECT MPI for medical reasons and CAD patient selection was made based on the previous medical records and written diagnosis of CAD. This made the CAD patient group a versatile group, which might have introduced some bias in the patient selection. However, the present data represent a picture of the actual population of subjects submitted to MPI.

\section{CONCLUSIONS}

A considerable part of CAD patients had significant LVMD. In this study, CABG or PCI did not independently predict dyssynchrony, but previous myocardial infarct, myocardial ischemia as well as large EDV did.

\section{NEW KNOWLEDGE GAINED}

LVMD is common in CAD patients and is associated with presence of both reversible and irreversible perfusion defects. This connection highlights the importance of detecting and treating of CAD to prevent possible upcoming heart failure.

\section{Disclosures}

The authors have no conflicts of interest.

\section{Funding}

Open access funding provided by University of Eastern Finland (UEF) including Kuopio University Hospital.

\section{Open Access}

This article is licensed under a Creative Commons Attribution 4.0 International License, which permits use, sharing, adaptation, distribution and reproduction in any medium or format, as long as you give appropriate credit to the original author(s) and the source, provide a link to the
Creative Commons licence, and indicate if changes were made. The images or other third party material in this article are included in the article's Creative Commons licence, unless indicated otherwise in a credit line to the material. If material is not included in the article's Creative Commons licence and your intended use is not permitted by statutory regulation or exceeds the permitted use, you will need to obtain permission directly from the copyright holder. To view a copy of this licence, visit http://creativecommons.org/licenses/by/4.0/.

\section{References}

1. Miyachi H, Yamamoto A, Otsuka T, et al. Relationship between left ventricular dyssynchrony and systolic dysfunction is independent of impaired left ventricular myocardial perfusion in heart failure: Assessment with $99 \mathrm{mTc}$-sestamibi gated myocardial scintigraphy. Int J Cardiol 2013;167:930-5.

2. Kirk JA, Kass DA. Electromechanical dyssynchrony and resynchronization of the failing heart. Circ Res 2013;113:765-76.

3. Park RC, Little WC, O'Rourke RA. Effect of alteration of left ventricular activation sequence on the left ventricular end-systolic pressure-volume relation in closed-chest dogs. Circ Res 1985;57:706-17.

4. Verberne HJ, Acampa W, Anagnostopoulos C, et al. EANM procedural guidelines for radionuclide myocardial perfusion imaging with SPECT and SPECT/CT: 2015 revision. Eur J Nucl Med Mol Imaging 2015;42:1929-40.

5. Henneman MM, Chen J, Ypenburg C, et al. Phase analysis of gated myocardial perfusion single-photon emission computed tomography compared with tissue doppler imaging for the assessment of left ventricular dyssynchrony. J Am Coll Cardiol 2007;49:1708-14

6. Bookers M. Quantitative gated SPECT-derived phase analysis on gated myocardial perfusion SPECT detects left ventricular dyssynchrony and predicts response to cardiac resynchronization therapy. J Nucl Med 2009;50:718-25.

7. Tavares A, Peclat T, Lima RS. Prevalence and predictors of left intraventricular dyssynchrony determined by phase analysis in patients undergoing gatedSPECT myocardial perfusion imaging. Int J Cardiovasc Imaging 2016;3:845.

8. Gimelli A, Liga R, Giorgetti A, Favilli B, Pasanisi EM, Marzullo P. Determinants of left ventricular mechanical dyssynchrony in patients submitted to myocardial perfusion imaging: A cardiac CZT study. J Nucl Cardiol 2016;23:728-36.

9. Huang W, Huang C, Lee C, Chen C, Hung G, Chen J. Relation of early post-stress left ventricular dyssynchrony and the extent of angiographic coronary artery disease. J Nucl Cardiol 2014;21:1048.

10. Li M, Li L, Wu W, Ran H, Zhang P. Left ventricular dyssynchrony in coronary artery disease patients without regional wall-motion abnormality: Correlation with gensini score. Echocardiography 2019;36:1689.

11. Azazy AS, Soliman M, Yaseen R, Mena M, Sakr H. Left ventricular dyssynchrony assessment using tissue synchronization imaging in acute myocardial infarction. Avicenna $\mathrm{J}$ Med 2019;9(2):48-54.

12. Zhang F, Tian Y, Yang W, et al. Myocardial perfusion imaging detects mechanical dyssynchrony in left ventricular infarcted and noninfarcted areas early after acute myocardial infarction in a porcine model. Nucl Med Commun 2019;40:115-23. 
13. Ludwig DR, Friehling M, Schelbert EB, Schwartzman D. Impact of scar on SPECT assay of left ventricular contraction dyssynchrony. Eur J Nucl Med Mol Imaging 2014;41:529-35.

14. Zhang Y, Chan AK, Yu C, et al. Left ventricular systolic asynchrony after acute myocardial infarction in patients with narrow QRS complexes. Am Heart J 2005;149:497-503.

15. Peix A, Cabrera LO, Padron K, et al. Association between non-perfusion parameters and presence of ischemia in gated-SPECT myocardial perfusion imaging studies. J Nucl Cardiol 2018;25:609-15.

16. Zafrir N. Left ventricular mechanical dyssynchrony in patients with coronary artery disease. J Nucl Cardiol 2016;24:491.

17. Germano G, Kavanagh PB, Waechter P, et al. A new algorithm for the quantitation of myocardial perfusion SPECT. I: Technical principles and reproducibility. J Nucl Med 2000;41:712-9.

18. Slomka PJ, Nishina H, Berman DS, et al. Automatic quantification of myocardial perfusion stress-rest change: A new measure of ischemia. J Nucl Med 2004;45:183-91.

19. Kang X, Berman DS, Van Train KF, et al. Clinical validation of automatic quantitative defect size in rest technetium-99m-sestamibi myocardial perfusion SPECT. J Nucl Med 1997;38:1441-6.

20. Hess PL, Shaw LK, Fudim M, Iskandrian AE, Borges-Neto S. The prognostic value of mechanical left ventricular dyssynchrony defined by phase analysis from gated single-photon emission computed tomography myocardial perfusion imaging among patients with coronary heart disease. J Nucl Cardiol 2016;24:482.

21. Van Kriekinge SD, Nishina H, Ohba M, Berman DS, Germano G. Automatic global and regional phase analysis from gated myocardial perfusion SPECT imaging: Application to the characterization of ventricular contraction in patients with left bundle branch block. J Nucl Med 2008;49:1790-7.

22. Hämäläinen $H$, Hedman M, Laitinen T, Hedman A, Kivela A, Laitinen T. Reference values for left ventricular systolic synchrony according to phase analysis of ECG-gated myocardial perfusion SPECT. Clin Physiol Funct Imaging 2018;38:38-45.

23. Wang J, Wang Y, Zhang X, Zhou R, Niu R, Lu P. Left ventricular systolic synchrony assessed by phase analysis of gated myocardial perfusion imaging in patients with old myocardial infarction. Zhonghua Xin Xue Guan Bing Za Zhi 2015;43:599-604.

24. Atchley AE, Kitzman DW, Whellan DJ, et al. Myocardial perfusion, function, and dyssynchrony in patients with heart failure: Baseline results from the single-photon emission computed tomography imaging ancillary study of the heart failure and A controlled trial investigating outcomes of exercise TraiNing (HFACTION) trial. Am Heart J 2009;158:S53-63.

25. Sobic-Saranovic DP, Pavlovic SV, Beleslin BD, et al. Site of myocardial infarction and severity of perfusion abnormalities impact on post-stress left ventricular function in patients with single-vessel disease: Gated single-photon emission computed tomography methoxyisobutylisonitrile study. Nucl Med Commun 2009;30:148-54.

26. Singh H, Patel CD, Sharma P, Naik N, Singh S, Narang R. Does perfusion pattern influence stress-induced changes in left ventricular mechanical dyssynchrony on thallium-201-gated SPECT myocardial perfusion imaging? J Nucl Cardiol 2014;22:36.

27. Aljaroudi W, Koneru J, Heo J, Iskandrian AE. Impact of ischemia on left ventricular dyssynchrony by phase analysis of gated single photon emission computed tomography myocardial perfusion imaging. J Nucl Cardiol 2011;18:36-42.

28. Mostafa SA, Mansour HA, Aboelazm TH, Elrabat KE, Sabry S. Impact of elective PCI on left intraventricular mechanical dyssynchrony in patients with chronic stable angina (tissue doppler study). Egypt Heart J 2014;67:259.

29. Inci S, Karakelleoglu S, Tas MH, et al. Acute effect of primary percutaneous coronary intervention on left ventricular dyssynchrony in ST-segment elevation myocardial infarction. Anadolu Kardiyol Derg 2014;14:591-8.

30. Siva Sankara C, Rajasekhar D, Vanajakshamma V, Praveen Kumar BS, Vamsidhar A. Prognostic significance of NT-proBNP, 3D LA volume and LV dyssynchrony in patients with acute STEMI undergoing primary percutaneous intervention. Indian Heart J 2015;67:318-27.

31. Ozdemir S, Yener AU, Barutcu A, Tan YZ, Celik F. The assessment of septal wall motion in patients undergoing CABG by myocardial perfusion-gated SPECT. Nucl Med Commun 2015;36:738.

32. Choi SH, Choi SI, Chun EJ, et al. Abnormal motion of the interventricular septum after coronary artery bypass graft surgery: Comprehensive evaluation with MR imaging. Korean J Radiol 2010;11:627-31.

33. Joshi SB, Salah AK, Mendoza DD, Goldstein SA, Fuisz AR, Lindsay J. Mechanism of paradoxical ventricular septal motion after coronary artery bypass grafting. Am J Cardiol 2009;103:2125 .

34. Aljaroudi W, Alraies MC, Brunken R, Cerquiera M, Jaber WA. Paradoxical septal motion from prior coronary artery bypass graft surgery does not impact left ventricular mechanical dyssynchrony by gated myocardial perfusion imaging. J Nucl Cardiol 2012;19:1190-7.

35. Park S, Cheon GJ, Paeng JC, et al. Phase analysis of gated myocardial perfusion single-photon emission computed tomography after coronary artery bypass graft surgery: Reflection of late reverse remodeling in patients with patent grafts after coronary artery bypass graft surgery. Nucl Med Commun 2016;37:1139-47.

Publisher's Note Springer Nature remains neutral with regard to jurisdictional claims in published maps and institutional affiliations. 